\title{
New Physics Contribution to Neutral Trilinear Gauge Boson Couplings
}

\author{
Sukanta Dutta ${ }^{1 *}$ Ashok Goyal ${ }^{2}+ \pm$ and Mamta1 1 \\ ${ }^{1}$ SGTB Khalsa College, University of Delhi. Delhi-11000\%. India. and \\ 2 Department of Physics \& Astrophysics, \\ University of Delhi. Delhi-11000\%. India.
}

\begin{abstract}
We study the one loop new physics effects to the CP even triple neutral gauge boson vertices $\gamma^{\star} \gamma Z, \gamma^{\star} Z Z, Z^{\star} Z \gamma$ and $Z^{\star} Z Z$ in the context of Little Higgs models. We compute the contribution of the additional fermions in Little Higgs models in the framework of direct product groups where $[S U(2) \times U(1)]^{2}$ gauge symmetry is embedded in $S U(5)$ global symmetry and also in the framework of simple group where $S U(N) \times U(1)$ gauge symmetry breaks down to $S U(2)_{L} \times U(1)$. We calculate the contribution of the fermions to these couplings when $T$ parity is invoked. In addition, we re-examine the MSSM contribution at the chosen point of SPS1a' and compare with the SM and Little Higgs models.

PACS numbers: 12.15.-y, 12.60.cn, 13.10.+q, 13.10.+q, 14.70.-c
\end{abstract}

Keywords: gauge boson couplings, one-loop, little Higgs

*Electronic address: Sukanta.Dutta@fnal.gov

$\dagger$ Electronic address: agoyal@iucaa.ernet.in

${ }^{\ddagger}$ Electronic address: mdahiya@physics.du.ac.in 


\section{INTRODUCTION}

Multiple gauge boson production channels at the Large Hadron Collider (LHC) provide a novel opportunity to probe the trilinear and quartic gauge boson couplings [1]. The proposed International Linear Collider (ILC) will be much more sensitive to these couplings due to its clean environment and fixed center of mass energy [2]. Availability of high luminosity in both these colliders gives us an unique facility to understand the non-Abelian gauge structure of the Standard Model (SM) and confront the presence of the new physics above the weak scale. The charged $W W \gamma$ and $W W Z$ couplings have been extensively studied and theoretical predictions in the context of SM and the minimal super-symmetric standard model (MSSM) have been made [3]. The neutral gauge-boson couplings $Z \gamma \gamma, Z Z \gamma$ and $Z Z Z$ which can be studied in $Z \gamma$ and $Z Z$ pair production in $e^{+} e^{-}$and in hadron colliders through $e^{+} e^{-} \rightarrow Z \gamma, Z Z$ and $q \bar{q} \rightarrow Z \gamma, Z Z$ respectively have been analyzed within the SM and MSSM [4, 5]. Recently Armillis et. al. [6] performed a detailed study of the trilinear gauge boson interactions with additional anomalous $U(1)$ 's, which arises in the construction of various string motivated and large extra dimension models. The model independent analysis of the neutral gauge boson couplings for hadron colliders exits in the literature [7] and has been also recently studied with the Tevatron Data [8]. Recent LEP studies on the triple gauge boson couplings have been made in references [9].

The forthcoming experiments at the LHC and proposed ILC offer the exciting prospect of probing physics beyond SM. In particular if super-symmetry exists in nature, there will be a real possibility of discovering super-symmetric partners. As is well known super symmetry (SUSY) provides an elegant solution to the Hierarchy problem in the SM by canceling the quadratic divergence arising in the Higgs mass due to the contributions of SM particles by the contributions from their super partners.

Recently there has been a proposal to consider Higgs fields as pseudo Nambu-Goldstone bosons of a global symmetry [10, 11, 12, 13, 14, 15] which is spontaneously broken at some high scale. The Higgs acquires mass through electro-weak symmetry breaking triggered by radiative corrections leading to a Coleman-Weinberg type of potential. Since the Higgs is protected by approximate global symmetry, it remains light and the quadratic contributions to its mass are canceled by the contributions of heavy gauge bosons and heavy fermionic states that are introduced in the model. If this little Higgs mechanism is realized in nature, it will be of great importance to verify it at the LHC. The realization of little Higgs mechanism discussed in the literature essentially fall into two classes [14]. The majority of implementations rely on the product group for the structure of gauge symmetry, its breaking pattern and the treatment of 
new heavy fermionic sector required to cancel the quadratic divergence contribution coming from the top quark. The second implementation has a simple group structure and has an additional model parameter. The Littlest Higgs model (LH) [11] is a minimal model of the product group class which accomplishes this task to one loop order within a minimal matter content. $\mathrm{SU}(3)$ simple group model [14, 15] is a representative model of the second class. The SU(3) gauge symmetry in this model forces the introduction of heavy partners with each $S U(2)_{L}$ fermion doublets of the SM. This model has an extra parameter. Both these models however, suffer from severe constraints [16, 17, 18] from precision electro-weak measurements which can be satisfied only by tuning the model parameters once again and thus introducing what is called a little hierarchy problem.

Motivated by these considerations, an implementation of a discrete symmetry called T-parity is proposed. T-parity explicitly forbids any tree-level contribution from the heavy mass states to observables involving only the SM particles. It also forbids the interactions that impart vev to triplet Higgs, thereby generating the corrections to precision electro-weak observables only

at the one loop level. In this little Higgs model with T-parity (LHT) [19] there are heavy T-odd partners of the SM gauge bosons and SM fermions called mirror fermions. In the top quark sector, the model incorporates two heavy T-even and T-odd top quarks in addition to the $\mathrm{T}$ even SM top quark, which are required for canceling the quadratically divergent contribution of the SM top quark to the Higgs mass. The LHT has rich phenomenology and the LHC has great potential to unravel it by directly observing the T-partners of the SM particles as well as by studying indirect phenomenological consequences [20, 21].

In this paper we study the CP conserving trilinear neutral gauge-boson couplings in little Higgs models and MSSM as discussed above. In section II, we evaluate one loop fermion contribution to these three point functions when one of the gauge bosons is off-shell and in section III, we analyze and interpret the numerical results.

\section{NEUTRAL GAUGE-BOSON COUPLINGS}

Bose-Einstein statistics render the three neutral gauge-boson couplings $\gamma \gamma Z, \gamma Z Z$ and $Z Z Z$ to vanish when all the three vector bosons are on shell. The most general CP conserving coupling of one off-shell boson $\mathrm{V} \equiv Z / \gamma$ to a pair of on-shell $Z \gamma$ and $Z Z$ gauge bosons (all incoming) can be written as (see Ref. [5])

$$
\begin{aligned}
& \Gamma_{\mathrm{V} Z \gamma}^{\mu \alpha \beta}\left(Q, p_{1}, p_{2}\right)=i\left[\mathcal{H}_{3}^{\mathrm{V}} \epsilon^{\mu \beta \alpha \eta} p_{2 \eta}+\frac{\mathcal{H}_{4}^{\mathrm{V}}}{M_{Z}^{2}}\left\{\epsilon^{\mu \beta \rho \eta} p_{2 \rho} Q_{\eta} Q^{\alpha}\right\}\right] \\
& \Gamma_{\mathrm{V} Z Z}^{\mu \alpha \beta}\left(Q, p_{1}, p_{2}\right)=i\left[\mathcal{F}_{5}^{\mathrm{V}} \epsilon^{\mu \alpha \beta \sigma}\left(p_{1}-p_{2}\right)_{\eta}\right]
\end{aligned}
$$


where the form factors $\mathcal{H}_{i}^{\mathrm{V}}$ and $\mathcal{F}_{5}^{\mathrm{V}}$ are related to those of reference [22] by

$$
\mathcal{H}_{i}^{\gamma}=\frac{Q^{2}}{m_{Z}^{2}} h_{i}^{\gamma}, \quad \mathcal{H}_{i}^{Z}=\frac{m_{Z}^{2}-Q^{2}}{m_{Z}^{2}} h_{i}^{Z} \text { and } \mathcal{F}_{5}^{\mathrm{V}}=-\frac{Q^{2}-m_{\mathrm{V}}^{2}}{m_{Z}^{2}} f_{5}^{V}
$$

Here $\Gamma_{\mathrm{V} V_{1} V_{2}}^{\mu \alpha \beta}\left(Q, p_{1}, p_{2}\right)$ represents the coupling of off-shell neutral gauge boson $V^{\mu}$ carrying momentum $Q$ with the bosons $V_{1}^{\alpha}$ and $V_{2}^{\beta}$ carrying momenta $p_{1}$ and $p_{2}$ respectively.

In the SM these couplings vanish at the tree level. These couplings can however, be generated at the loop level Fig. (5). On account of the totally antisymmetric nature of $\epsilon^{\mu \alpha \beta \sigma}$, these couplings can never be generated by scalars and vector-bosons running in the loop. Thus fermions running in the loop with one axial and two vector-couplings or all the three axial-couplings at the vertices can generate such couplings. Further at the one loop level, the couplings $\mathcal{H}_{4}^{\gamma}$ and $\mathcal{H}_{4}^{Z}$ are not generated i.e.

$$
\mathcal{H}_{4}^{\gamma}=\mathcal{H}_{4}^{Z}=0
$$

Thus the only couplings likely to appear at one-loop are $\mathcal{F}_{5}^{\gamma, Z}$ and $\mathcal{H}_{3}^{\gamma, Z}$. These couplings can in general be complex quantities. However, they pick up imaginary contribution only when $Q^{2}$ crosses the threshold for fermion pair production (i.e. $Q^{2}>4 m_{f}^{2}$ ) for time-like $Q^{2}$ or when $M_{Z}^{2}$ exceeds this threshold (i.e. $M_{Z}>2 m_{f}$ ) for space-like $Q^{2}$.

In order to evaluate these couplings, we write the interactions of the vector boson $\mathrm{V} \equiv \gamma, Z$ with fermions in the standard notation

$$
\mathcal{L}_{\text {int }}=\bar{f}_{i} \gamma_{\mu}\left[g_{L_{i j}}^{\mathrm{V}} P_{L}+g_{R_{i j}}^{\mathrm{V}} P_{R}\right] f_{j} \mathrm{~V}^{\mu}
$$

For $\mathrm{V} \equiv \gamma$, we have $g_{L_{i j}}=g_{R_{i j}}=\delta_{i j}$ e $q_{i}, q_{i}$ being the charge of fermion $f_{i}$. For $\mathrm{V}=Z$, the couplings $g_{L}$ and $g_{R}$ in various models are listed in Table IV of the Appendix. In the absence of any CP violating interactions, all these couplings are real and $g_{L, R_{i j}}=g_{L, R_{j i}}$ because of hermiticity.

Using the notation of Passarino-Veltman (PV) functions, the contribution of the fermionic triangle graphs to the trilinear vector boson couplings $\mathcal{F}_{5}^{\gamma, Z}$ and $\mathcal{H}_{3}^{\gamma, Z}$ can be expressed in terms of scalar PV functions as given in the Appendix.

\section{A. SM contribution}

The contribution to the trilinear neutral gauge couplings in the SM arise from the three families of quarks and leptons. The anomaly cancellation ensures that all the couplings go to zero for $Q^{2}$ much larger than the fermion pair production threshold. It is obvious that of all the thresholds (at $Q^{2}=4 M_{f}^{2}$ ), the largest contribution comes from the heaviest fermion loop. The couplings $\mathcal{F}_{5}^{\gamma}$ and $\mathcal{H}_{3}^{\gamma, Z}$ get contributions only from the charged fermions, whereas $\mathcal{F}_{5}^{Z}$ 
receives contributions from the neutrinos as well. Note that in SM, the same fermion runs in all the three sides of triangle loop as there is no mixed coupling of neutral boson with different fermions.

\section{B. MSSM}

The MSSM contribution to the trilinear neutral gauge couplings has been calculated in the references [4, 5]. Charginos contribute to all the four anomalous couplings where as the neutralinos contribute only to the $\mathcal{F}_{5}^{Z}$. We re-calculate the MSSM contribution in the light of the reference point SPS1a' which is defined at a characteristic scale of $1 \mathrm{TeV}$ with its origin in minimal super-gravity (mSUGRA) [25]. The root GUT scale mSUGRA parameters in this reference point SPS1a' are the gaugino mass $M_{1 / 2}=250 \mathrm{GeV}$, the universal scalar mass $M_{0}=70$ $\mathrm{GeV}$, the trilinear coupling $A_{0}=-300 \mathrm{GeV}, \tan \beta(\tilde{M})=10$ and $\operatorname{sign}(\mu)=+1$. Extrapolating these parameters to $\tilde{M}=1 \mathrm{TeV}$ generates the MSSM Lagrangian parameters. The relevant evolved MSSM parameters for our calculations are the Higgs mixing parameter $\mu=396 \mathrm{GeV}$ and $M_{2}=193.2 \mathrm{GeV}$.

\section{LH contribution}

In the little Higgs models, the Higgs bosons are realized as pseudo-Goldstone bosons. The generic structure of little Higgs models is a global symmetry broken at a high (TeV) scale $f$. At this scale there are new gauge bosons, scalars and fermions responsible for the cancellation

of the quadratic divergent one loop contributions to the Higgs boson mass from the SM gauge bosons, Higgs self interactions and from the top quark respectively. The Littlest Higgs model accomplishes this task with the minimal matter content. In this model $[S U(2) \times U(1)]^{2}$ gauge symmetry is embedded in an $S U(5)$ global symmetry. The gauge symmetry is broken down to the $\mathrm{SM} S U(2) \times U(1)$ gauge symmetry by a single vacuum condensate $f \approx 1 \mathrm{TeV}$. The new fermionic degrees of freedom in the Littlest Higgs model are in the heavy quark sector and consist of a pair of vector-like $S U(2)$-singlet quarks that couple to the top sector. The resultant top sector consists of a top quark $t$ and its heavy partner $\mathrm{T}$ whose masses and couplings are given in terms of model dependent parameters by

$$
\begin{aligned}
m_{t} & =\frac{\lambda_{1} \lambda_{2}}{\sqrt{\lambda_{1}^{2}+\lambda_{2}^{2}}} v \\
M_{T} & =\sqrt{\lambda_{1}^{2}+\lambda_{2}^{2}} f=\frac{1}{\sqrt{X_{L}\left(1-X_{L}\right)}} \frac{m_{t}}{v} f
\end{aligned}
$$


where $X_{L}=\lambda_{1}^{2} /\left(\lambda_{1}^{2}+\lambda_{2}^{2}\right), \lambda_{1}$ and $\lambda_{2}$ being the couplings that appear in the heavy quark sector of the interaction lagrangian.

\begin{tabular}{|c|c|c|c|c|c|c|}
\hline $\begin{array}{c}\text { scale } \\
(\text { in } \mathrm{TeV})\end{array}$ & $\begin{array}{c}m_{T} \\
\text { (in } \mathrm{GeV})\end{array}$ & $\sqrt{Q^{2}}$ & $\begin{array}{c}\mathcal{H}_{3}^{\gamma} \\
\left(10^{-4}\right)\end{array}$ & $\begin{array}{c}\mathcal{H}_{3}^{Z} \\
\left(10^{-4}\right)\end{array}$ & $\begin{array}{c}\mathcal{F}_{5}^{\gamma} \\
\left(10^{-4}\right)\end{array}$ & $\begin{array}{c}\mathcal{F}_{5}^{Z} \\
\left(10^{-4}\right)\end{array}$ \\
\hline 0.5 & 711.4 & $\begin{array}{c}2 m_{t} \\
m_{t}+M_{T} \\
2 M_{T}\end{array}$ & $\begin{array}{c}-89.34-\iota 0.0130 \\
0.9154-\iota 28.02 \\
3.710-\iota 14.24\end{array}$ & $\begin{array}{c}25.49+0 \iota \\
5.388+\iota 7.730 \\
-0.3113+\iota 6.901\end{array}$ & $\begin{array}{c}-27.65+0 \iota \\
-1.313-\iota 9.229 \\
-0.0809-\iota 5.481\end{array}$ & $\begin{array}{c}-18.06+0 \iota \\
-8.534-\iota 11.50 \\
1.717-\iota 10.66\end{array}$ \\
\hline 1.0 & 1422.8 & $\begin{array}{c}2 m_{t} \\
m_{t}+M_{T} \\
2 M_{T}\end{array}$ & $\begin{array}{c}-92.97-\iota 0.0147 \\
4.6901-\iota 12.51 \\
2.976-\iota 5.004\end{array}$ & $\begin{array}{c}28.46+\iota 0.0003 \\
-0.1816+\iota 3.951 \\
-0.842+\iota 2.255\end{array}$ & $\begin{array}{c}-30.40+0 \iota \\
1.152-\iota 4.267 \\
0.6727-\iota 1.942\end{array}$ & $\begin{array}{c}-19.98+\iota 0.0002 \\
-0.5294-\iota 6.851 \\
1.710-\iota 3.926\end{array}$ \\
\hline 2.0 & 2845.5 & $\begin{array}{c}2 m_{t} \\
m_{t}+M_{T} \\
2 M_{T}\end{array}$ & $\begin{array}{c}-93.87-\iota 0.0152 \\
3.039--\iota 4.590 \\
1.396-\iota 1.587\end{array}$ & $\begin{array}{c}29.26+\iota 0.0008 \\
-0.7061+\iota 1.501 \\
-0.4402+\iota 0.6802\end{array}$ & $\begin{array}{c}-31.22+0 \iota \\
0.9257-\iota 1.573 \\
0.3927-\iota 0.6076\end{array}$ & $\begin{array}{c}-20.57+\iota 0.0002 \\
0.9056-\iota 2.891 \\
0.8645-\iota 1.2824\end{array}$ \\
\hline 3.0 & 4268.3 & $\begin{array}{c}2 m_{t} \\
m_{t}+M_{T} \\
2 M_{T}\end{array}$ & $\begin{array}{l}-94.04-\iota 0.0152 \\
0.8188-\iota 0.7906 \\
1.9801-\iota 2.4200\end{array}$ & $\begin{array}{c}29.41+0 \iota \\
0.2639+\iota 0.3314 \\
-0.5342+\iota 0.7975\end{array}$ & $\begin{array}{c}-31.37+0 \iota \\
0.2422-\iota 0.2998 \\
0.6259-\iota 0.8290\end{array}$ & $\begin{array}{c}-20.68+0 \iota \\
0.5222 \iota 0.6467 \\
0.7985-\iota 1.6052\end{array}$ \\
\hline
\end{tabular}

TABLE I: The values of various triple neutral gauge boson couplings in LH Model (written as complex numbers) at some typical $\sqrt{Q^{2}}$ (where peaks are expected) for different values of symmetry breaking scale $f$. All values correspond to $r=\lambda_{1} / \lambda_{2}=1$ and $m_{t}=175 \mathrm{GeV}$. The values are written in units of $10^{-4}$. Note that the lower values of the scale correspond only to the Littlest Higgs Model with T-Parity (the couplings in the two models being the same up to $\mathcal{O}(v / f)$ ) with $m_{T_{+}}=m_{T}$.

The interactions of the left and right handed fermions with the $Z$ boson in this model can be found in the reference [23] and can also be realized from the LHT couplings given in the Table IV by retaining only the leading terms in $v / f$ because of the large scale factor requirement from precision electro-weak data.

The second class of little Higgs models feature a simple group that contains an $S U(N) \times U(1)$ gauge symmetry that is broken down to $S U(2)_{L} \times U(1)$, giving rise to a set of $\mathrm{TeV}$-scale gauge bosons. The two gauge couplings of $S U(N) \times U(1)$ are fixed in terms of two SM gauge couplings, leaving no free parameters in the gauge sector. Furthermore, due to enlarged $S U(N)$ gauge symmetry, all fermionic SM representations are extended to transform as fundamental or conjugate representations of $S U(N)$. This gives rise to additional heavy fermions in all the three quark and lepton sectors. The simplest realization of this simple group class is the $S U(3)$ simple gauge model [14, 15] with anomaly-free embedding of extra fermions. The expansion of 
the $S U(2)_{L}$ gauge group to $S U(3)$ requires introduction of heavy fermions associated with each $S U(2)_{L}$ doublet of the SM. The first two generations of quarks are enlarged to contain the new TeV-scale D and $\mathrm{S}$ quarks of charge $-1 / 3$ that are $\overline{3}$ representations of $S U(3)$. The quarks of the third generation and three generation of leptons are put in the 3 representation of $S U(3)$. The electric charge of the heavy third generation quark $\mathrm{T}$ is $+2 / 3$ and all the heavy leptons $N_{i}$ of three generations are electrically neutral. The masses of these heavy fermions are given in terms the parameters of the model, namely,

$$
\begin{aligned}
M_{T} & =\sqrt{\lambda_{1}^{2} c_{\beta}^{2}+\lambda_{2}^{2} s_{\beta}^{2}} f=\sqrt{2} \frac{t_{\beta}^{2}+X_{\lambda}^{2}}{\left(1+t_{\beta}^{2}\right) X_{\lambda}} \frac{m_{t}}{v} f \\
M_{D, S} & =s_{\beta} \lambda_{D, S} f \\
M_{N_{i}} & =s_{\beta} \lambda_{N_{i}} f
\end{aligned}
$$

where $t_{\beta} \equiv \tan \beta=f_{2} / f_{1}$ is an additional parameter in the simple $S U(3)$ model and $X_{L}=$ $\lambda_{1}^{2} /\left(\lambda_{1}^{2}+\lambda_{2}^{2}\right)$. in $S U(3)$ simple group, $f$ is defined as $f \equiv \sqrt{f_{1}^{2}+f_{2}^{2}}$. In these expressions effect of light quark masses is neglected and the neutrinos are taken to be mass less. Constraints from electro-weak precision measurements require the breaking scale $f$ to be greater than $5 \mathrm{TeV}$ in the Littlest Higgs Model. This constraint can however be brought down to about $2-3 \mathrm{TeV}$ ( See for example Ref. [23] ). In the anomaly free $S U(3)$-simple group [18], the constraint on the scale is $f>3.9 \mathrm{TeV}$ for $t_{\beta}=3$. The scale $f$ can only be marginally brought down by slightly different realization [24]. In these two classes of models $T$ mass has a lower bound given by

$$
M_{T} \geq 2 \frac{m_{t}}{v} f \approx \sqrt{2} f \text { for } \lambda_{1}=\lambda_{2}
$$

in the Littlest Higgs model and

$$
M_{T} \geq 2 \sqrt{2} s_{\beta} c_{\beta} \frac{m_{t}}{v} f \approx f \sin (2 \beta) \text { for } \frac{\lambda_{1}}{\lambda_{2}}=\tan \beta
$$

in the $S U(3)$ simple group model.

We calculate the contributions to the trilinear gauge boson couplings in the LH model. Unlike SM, here the contribution to these couplings also comes from the triangle graph with both the SM top $t$ and heavy top $T$ simultaneously present in the loop because of the presence of $\bar{T} Z t$ coupling. The couplings relevant for our study can be read out from the Table IV of the Appendix.

In Table I, we give the values of real and imaginary parts of all four trilinear neutral gauge couplings as a function of symmetry breaking scale $f$ for fixed ratio $r=\frac{\lambda_{1}}{\lambda_{2}}=1$. The values are given for some typical values of $\sqrt{Q^{2}}$ where theoretical peaks are expected. It is worthwhile to mention once again, that the entries corresponding to the large values for the scale $f$ say, $\sim 2-3 \mathrm{TeV}$ corresponds to both the little Higgs Model with and without T Parity. However, 


\begin{tabular}{|c|c|c|c|c|}
\hline $\begin{array}{c}\sqrt{Q^{2}} \\
\text { (in } \mathrm{TeV})\end{array}$ & $\begin{array}{c}\mathcal{H}_{3}^{\gamma} \\
\left(10^{-4}\right)\end{array}$ & $\begin{array}{c}\mathcal{H}_{3}^{Z} \\
\left(10^{-4}\right)\end{array}$ & $\begin{array}{c}\mathcal{F}_{5}^{\gamma} \\
\left(10^{-4}\right)\end{array}$ & $\begin{array}{c}\mathcal{F}_{5}^{Z} \\
\left(10^{-4}\right)\end{array}$ \\
\hline $2 m_{t}$ & $-94.17-\iota 0.0158$ & $29.53+0 \iota$ & $-31.50+\iota 0.0149$ & $-22, .42+\iota 0.0254$ \\
\hline$m_{t}+M_{T}$ & $4.533-\iota 9.136$ & $-1.487+\iota 3.008$ & $1.757-\iota 2.802$ & $-0.1062-\iota 4.751$ \\
\hline $2 M_{T}$ & $2.582-\iota 3.448$ & $-2.417-\iota 0.0712$ & $0.5535-\iota 0.677$ & $0.9483+\iota 0.9624$ \\
\hline$M_{U}$ & $3.146-\iota 4.660$ & $-2.503+\iota 2.036$ & $1.146-\iota 1.152$ & $1.699-\iota 2.449$ \\
\hline $2 M_{U}$ & $1.372-\iota 1.455$ & $0.1424-\iota 1.523$ & $2.947-\iota 0.191$ & $-3.151+\iota 2.236$ \\
\hline
\end{tabular}

TABLE II: The values of various couplings (written as complex numbers) at some typical $\sqrt{Q^{2}}$ (where peaks are expected) in the $S U(3)$ simple model with anomaly free embedding. All values correspond to $\tan \beta=r=3$, scale $f=3 \mathrm{TeV}$ and $m_{t}=175 \mathrm{GeV}$. At these values of parameters, the mass of heavy top is $M_{T}=1.8 \mathrm{TeV}$ and masses of all other heavy fermions have been taken to be $M_{i}=3 \mathrm{TeV}$.

the results derived from the lower values of the scale $f$ corresponds only to the Little Higgs Model with T parity (discussed later in the following section) as the measured value of precision observables forbids lower value of $f$ in the littlest Higgs model. It is to be noted that up to the leading order in $x=\mathcal{O}(v / f)$, the couplings in both the models are same and for large $f$ the higher order terms in $x$ may be easily neglected.

We also calculate the contributions to the trilinear gauge boson couplings in the anomaly free $\mathrm{SU}(3)$ simple group model. A new feature in this model is the contribution from mixed $t$ and $T$, mixed SM and TeV range quarks of the first two generations and the mixed neutrino and $\mathrm{TeV}$ mass heavy neutrinos $\left(N_{i}\right)$ of all the three generations in the triangle loop. However, the pure $T$ quark loop, pure TeV mass quark loop of first two generations and TeV mass heavy neutrinos of three generations do not contribute to $\mathcal{F}_{5}^{Z}$ in the model. This is clear from the couplings of Z-boson to various new fermions (Table IV).

The Table II lists the values of the couplings for the anomaly free SU(3) simple group Model. All values correspond to $\tan \beta=r=3$, scale $f=3 \mathrm{TeV}$ and $m_{t}=175 \mathrm{GeV}$. At these values of the parameters, the mass of heavy top is $M_{T}=1.8 \mathrm{TeV}$ and masses of all other heavy fermions have been taken to be $M_{i}=3 \mathrm{TeV}$. As expected, the threshold values at $2 m_{t}$ has roughly the same magnitude as that in the SM. At higher $\sqrt{Q^{2}}$, the effect of new heavy fermions shows up but the threshold values are an order of magnitude lower than that at the $2 m_{t}$ threshold. However, at these $\sqrt{Q^{2}}$, the SM contribution is negligible. In Fig. 1 we have shown this behavior of couplings as a function of $\sqrt{Q^{2}}$. The values of various parameters are the same as given in Table II but the mass of heavy fermions $U$ and $N$ which are taken to be $2 \mathrm{TeV}$ each in the figure. 

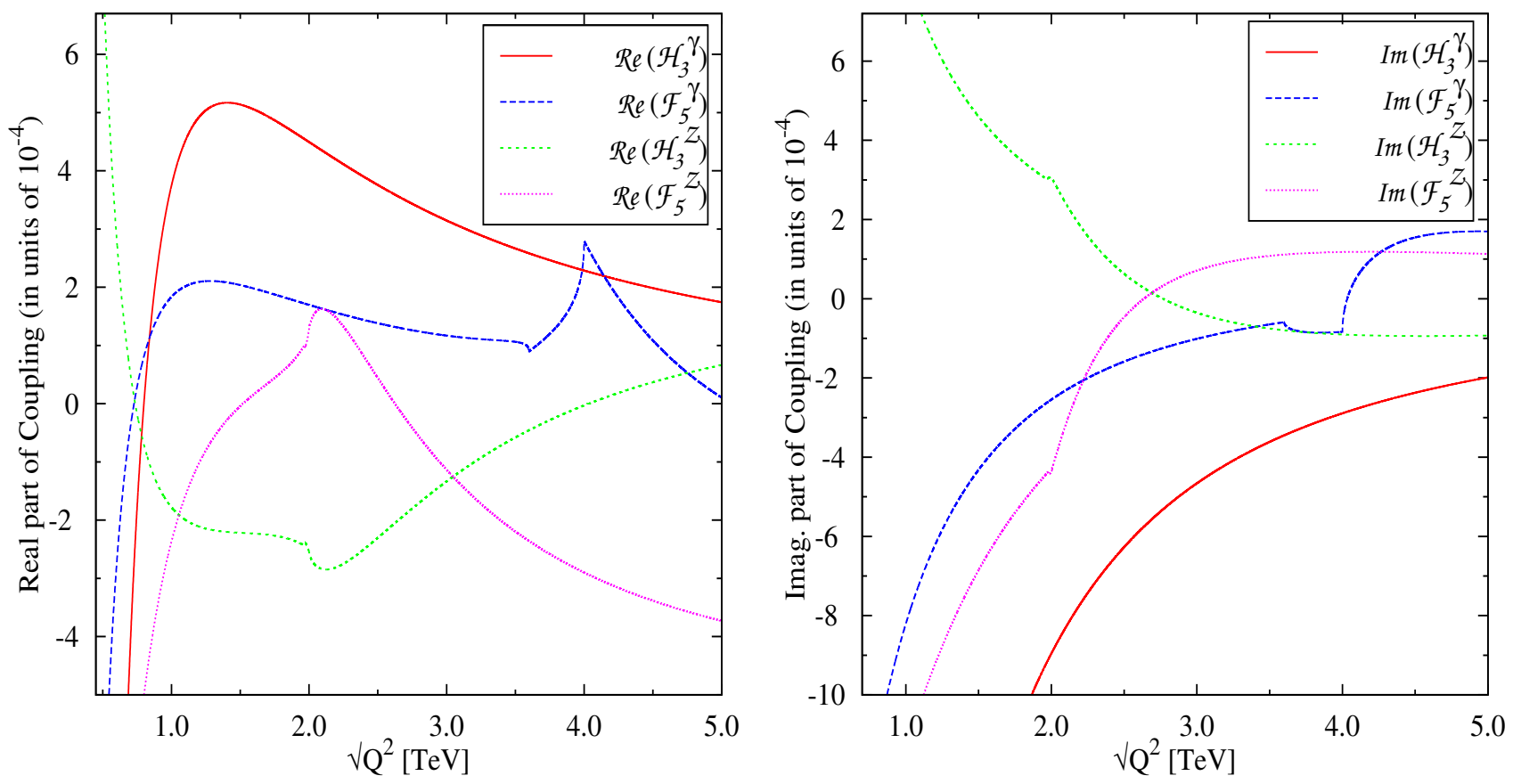

FIG. 1: $\sqrt{Q^{2}}$-variation in the range $1-5 \mathrm{TeV}$ of the 1) real and 2) imaginary parts of the couplings in $S U(3)$ simple Little Higgs Model with anomaly free embedding for $\tan \beta=r=3, f=3 \mathrm{TeV}$. With this choice of parameters the mass of heavy top $M_{T}=1.8 \mathrm{TeV}$ while the masses of all other heavy fermions are fixed at $2 \mathrm{TeV}$.

\section{LHT contribution}

In LHT as discussed in the Introduction, the T-odd heavy ( $\mathrm{TeV}$ mass) fermions called mirror fermions couple vectorially to $Z_{\mu}$. Further, because of $T$-parity conservation there is no coupling between $Z_{\mu}$ and T-odd and $T$-even fermions i.e. the coupling $Z \bar{f}_{+} f_{-}=0$ Thus the mirror fermions do not contribute to the trilinear neutral gauge boson couplings. The $T$-even partner of the top quark $T_{+}$however, has both axial and vector couplings with $Z_{\mu}$ and hence contributes to the triangle loop. The top quark masses in this model are given by

$$
\begin{aligned}
m_{t} & =\frac{\lambda_{1} \lambda_{2}}{\sqrt{\lambda_{1}^{2}+\lambda_{2}^{2}}} v\left\{1+\frac{v^{2}}{f^{2}}\left(-\frac{1}{3}+\frac{1}{2} X_{L}\left(1-X_{L}\right)\right)\right\} \\
M_{T} & =\frac{m_{t}}{\sqrt{X_{L}\left(1-X_{L}\right)}} \frac{f}{v}\left\{1+\frac{v^{2}}{f^{2}}\left(\frac{1}{3}-X_{L}\left(1-X_{L}\right)\right)\right\}
\end{aligned}
$$

In addition to the contribution from SM fermions in the loop, we now get additional contri- 


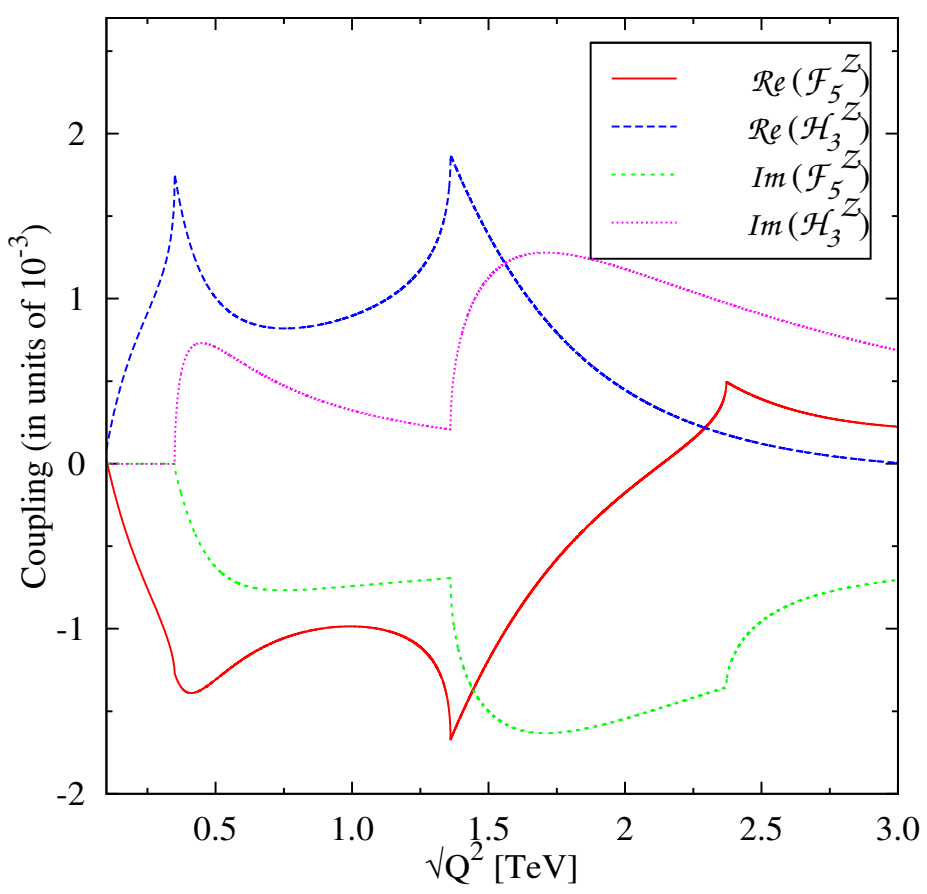

FIG. 2: $\sqrt{Q^{2}}$-variation of real and imaginary parts of $\mathcal{H}_{3}^{Z}$ and $\mathcal{F}_{5}^{Z}$ in the range $0-3 \mathrm{TeV}$ in Little Higgs Model with T-parity for $r=3$ and $f=0.5 \mathrm{TeV}$. With this choice of parameters the mass of T-even top, $M_{T_{+}}=1.186 \mathrm{TeV}$.

butions from heavy $T$-even partner and the top quark as well as contributions from the triangle loops with mixed contributions from $t$ and $T+$ quarks. The relevant fermion couplings with $Z$ boson are given in table IV of the appendix.

The Table [II lists the values of the couplings in the Little Higgs Model for different values of the $r$ ratio and at some typical values of $\sqrt{Q^{2}}$. All the values are for symmetry breaking scale $f=500 \mathrm{GeV}$ and are given in units of $10^{-4}$. This table also gives the value of heavy T-even top mass for different ratios. As expected for the same value of $f$, the mass $m_{T_{+}}$is the same for the ratio $r$ and $1 / r$. It may be mentioned that for higher ratios, a very interesting behavior is shown by the couplings $\mathcal{H}_{3}^{Z}$ and $\mathcal{F}_{5}^{Z}$. Not only the imaginary part becomes appreciable at high $\sqrt{Q^{2}}$ but also the threshold values of the couplings at $\sqrt{Q^{2}}=m_{t}+m_{T_{+}}$are higher than those at $\sqrt{Q^{2}}=2 m_{t}$ and are comparable to the SM values. This is clearly brought about in Fig. 2 where we have plotted the real and imaginary parts of the couplings $\mathcal{H}_{3}^{Z}$ and $\mathcal{F}_{5}^{Z}$ for $\sqrt{Q^{2}}$ up to $3 \mathrm{TeV}$, scale $f=0.5 \mathrm{TeV}$ and the ratio $r=3$. 


\begin{tabular}{|c|c|c|c|c|c|c|}
\hline ratio & $\begin{array}{c}M_{T_{+}} \\
(\text {in } \mathrm{GeV})\end{array}$ & $\sqrt{Q^{2}}$ & $\begin{array}{c}\mathcal{H}_{3}^{\gamma} \\
\left(10^{-4}\right)\end{array}$ & $\begin{array}{c}\mathcal{H}_{3}^{Z} \\
\left(10^{-4}\right)\end{array}$ & $\begin{array}{c}\mathcal{F}_{5}^{\gamma} \\
\left(10^{-4}\right)\end{array}$ & $\begin{array}{c}\mathcal{F}_{5}^{Z} \\
\left(10^{-4}\right)\end{array}$ \\
\hline 0.5 & 889.2 & $\begin{array}{c}2 m_{t} \\
m_{t}+M_{T_{+}} \\
2 M_{T_{+}}\end{array}$ & $\begin{array}{c}-93.40-\iota 0.0149 \\
4.068-\iota 22.963 \\
4.595-\iota 10.66\end{array}$ & $\begin{array}{c}28.85+0 \iota \\
-0.705+\iota 7.341 \\
-1.499+\iota 3.875\end{array}$ & $\begin{array}{c}-30.83+0 \iota \\
1.413-\iota 7.612 \\
1.441-\iota 3.666\end{array}$ & $\begin{array}{c}-20.29+0 \iota \\
-1.601-\iota 10.82 \\
1.574-\iota 6.475\end{array}$ \\
\hline 1.0 & 711.4 & $\begin{array}{c}2 m_{t} \\
m_{t}+M_{T_{+}} \\
2 M_{T_{+}}\end{array}$ & $\begin{array}{c}-89.34-\iota 0.0130 \\
0.9154-\iota 28.02 \\
3.710-\iota 14.24\end{array}$ & $\begin{array}{c}25.49+0 \iota \\
5.388+\iota 7.730 \\
-0.3113+\iota 6.901\end{array}$ & $\begin{array}{c}-27.65+0 \iota \\
-1.313-\iota 9.229 \\
-0.0809-\iota 5.481\end{array}$ & $\begin{array}{c}-18.06+0 \iota \\
-8.534-\iota 11.50 \\
1.717-\iota 10.66\end{array}$ \\
\hline 2.0 & 889.2 & $\begin{array}{c}2 m_{t} \\
m_{t}+M_{T_{+}} \\
2 M_{T_{+}}\end{array}$ & $\begin{array}{c}-81.80-\iota 0.0094 \\
0.7583-\iota 19.59 \\
1.116-\iota 9.099\end{array}$ & $\begin{array}{l}19.84-\iota 0.0054 \\
14.88+\iota 3.813 \\
1.424+\iota 9.069\end{array}$ & $\begin{array}{c}22.00+0 \iota \\
-4.727-\iota 7.226 \\
-2.866-\iota 5.549\end{array}$ & $\begin{array}{l}-14.31+\iota 0.0002 \\
-15.09-\iota 8.566 \\
-5.549-\iota 13.21\end{array}$ \\
\hline 3.0 & 1185.6 & $\begin{array}{c}2 m_{t} \\
m_{t}+M_{T_{+}} \\
2 M_{T_{+}}\end{array}$ & $\begin{array}{c}-78.51-\iota 0.0079 \\
0.4505-\iota 13.03 \\
-0.623-\iota 5.49\end{array}$ & $\begin{array}{l}17.58-\iota 0.0068 \\
18.70+\iota 2.076 \\
1.759+\iota 9.726\end{array}$ & $\begin{array}{c}-19.40+0 \iota \\
-6.278-\iota 6.100 \\
-3.972-\iota 5.508\end{array}$ & $\begin{array}{c}-12.69+\iota 0.0002 \\
-16.71-\iota 6.937 \\
4.953-\iota 13.55\end{array}$ \\
\hline 4.0 & 1511.7 & $\begin{array}{c}2 m_{t} \\
m_{t}+M_{T_{+}} \\
2 M_{T_{+}}\end{array}$ & $\begin{array}{c}-77.05-\iota 0.0072 \\
0.2019-\iota 9.182 \\
-1.712-\iota 3.614\end{array}$ & $\begin{array}{l}16.60-\iota 0.0074 \\
20.18+\iota 1.320 \\
1.824+\iota 9.939\end{array}$ & $\begin{array}{c}-18.16+0 \iota \\
-7.086-\iota 5.541 \\
-4.461-\iota 5.508\end{array}$ & $\begin{array}{c}-11.95+\iota 0.0002 \\
-17.0-\iota 6.011 \\
5.479-\iota 13.48\end{array}$ \\
\hline 5.0 & 1849.6 & $\begin{array}{c}2 m_{t} \\
m_{t}+M_{T_{+}} \\
2 M_{T_{+}}\end{array}$ & $\begin{array}{c}-76.30-\iota-0.0068 \\
-0.843-\iota 6.819 \\
-2.416-\iota 2.558\end{array}$ & $\begin{array}{c}16.10-\iota 0.0077 \\
20.78+\iota 0.9261 \\
1.841+\iota 10.01\end{array}$ & $\begin{array}{c}-17.49+0 \iota \\
-7.562-\iota 5.220 \\
-4.717-\iota 5.511\end{array}$ & $\begin{array}{c}-11.56+\iota 0.0002 \\
-17.14-\iota 5.422 \\
5.727-\iota 13.34\end{array}$ \\
\hline
\end{tabular}

TABLE III: The values of various couplings (written as complex numbers) at some typical $\sqrt{Q^{2}}$ (where peaks are expected) for different values of $r=\lambda_{1} / \lambda_{2}$ in the LHT Model. All values correspond to symmetry breaking scale $f=500 \mathrm{GeV}$ and $m_{t}=175 \mathrm{GeV}$.

\section{RESULTS AND DISCUSSION}

We calculate the one-loop contribution to the CP-conserving trilinear neutral gauge boson couplings in SM, MSSM and the two classes of Little Higgs Models for various parameters of the models. The $\sqrt{Q^{2}}$ variation of the real and imaginary parts of the couplings in all the four models is shown in Fig. 3 and 4 . Values at some typical $\sqrt{Q^{2}}$ are also given in the Tables [1, II] and III for different values of parameters of the model for the Little Higgs Model with T-parity and for the $\mathrm{SU}(3)$ Model with anomaly free embedding. Certain features are common to all these graphs which we note here. All couplings vanish asymptotically for large $\sqrt{Q^{2}}$ compared to the highest fermion mass in the theory. This is ensured by the anomaly cancellation in the models considered. The relative importance of the real and imaginary parts of the couplings is 

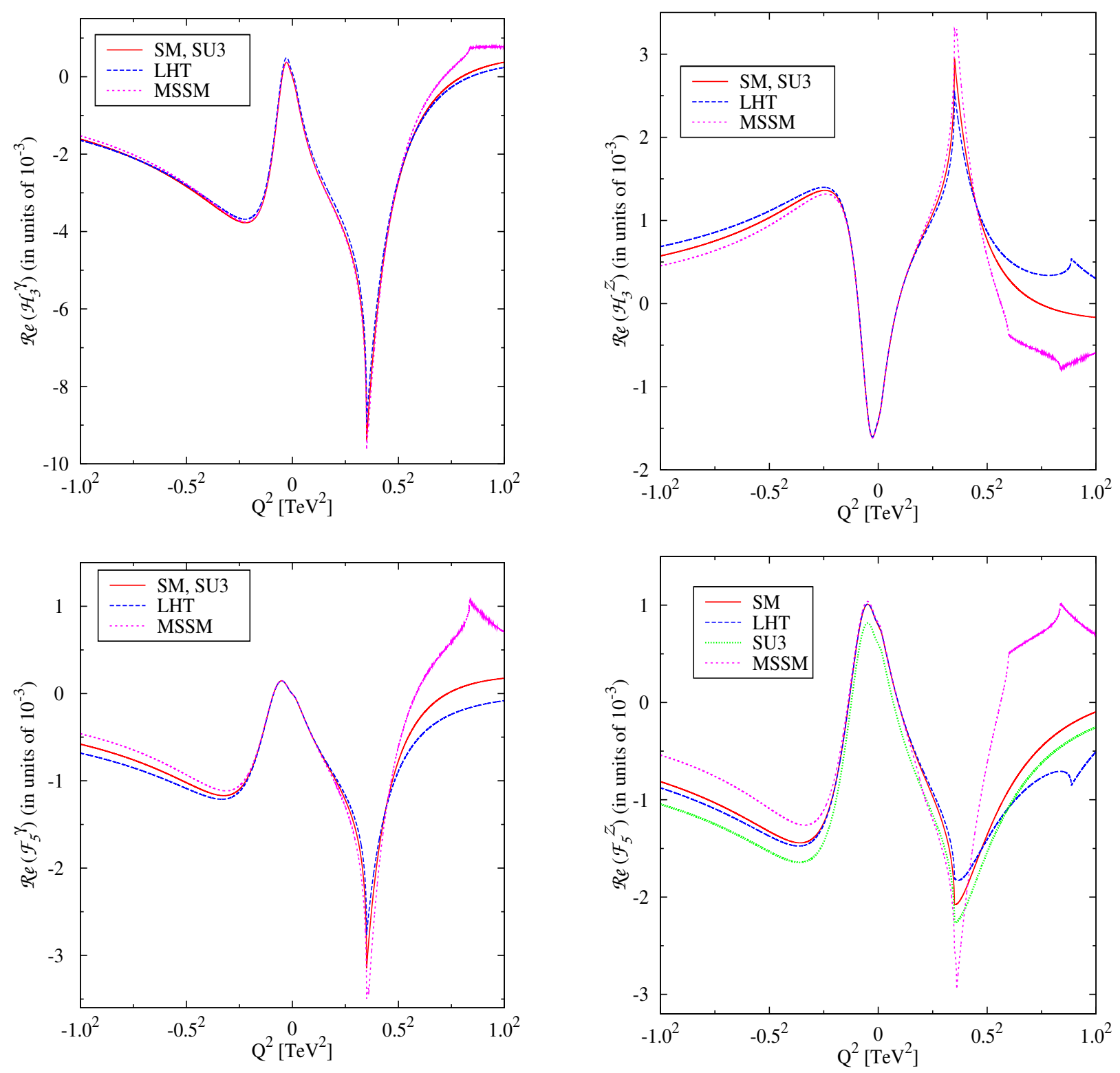

FIG. 3: $\sqrt{Q^{2}}$-variation of the real parts of the couplings in various models for model parameter values $f=500 \mathrm{GeV}, r=1$ for LHT and $f=3 \mathrm{TeV}, t_{\beta}=3$ and masses of all heavy fermions, $M_{i}=2 \mathrm{TeV}$ for the SU(3) Model. MSSM parameters are as discussed in the text.

strongly energy dependent. As expected and explained in Sec. II, below the $2 m_{t}$ threshold, the imaginary parts of all the couplings are negligible. At and above this threshold the imaginary parts become comparable or even dominant in comparison to the real parts. This behavior is shared by the couplings in all the models considered. 

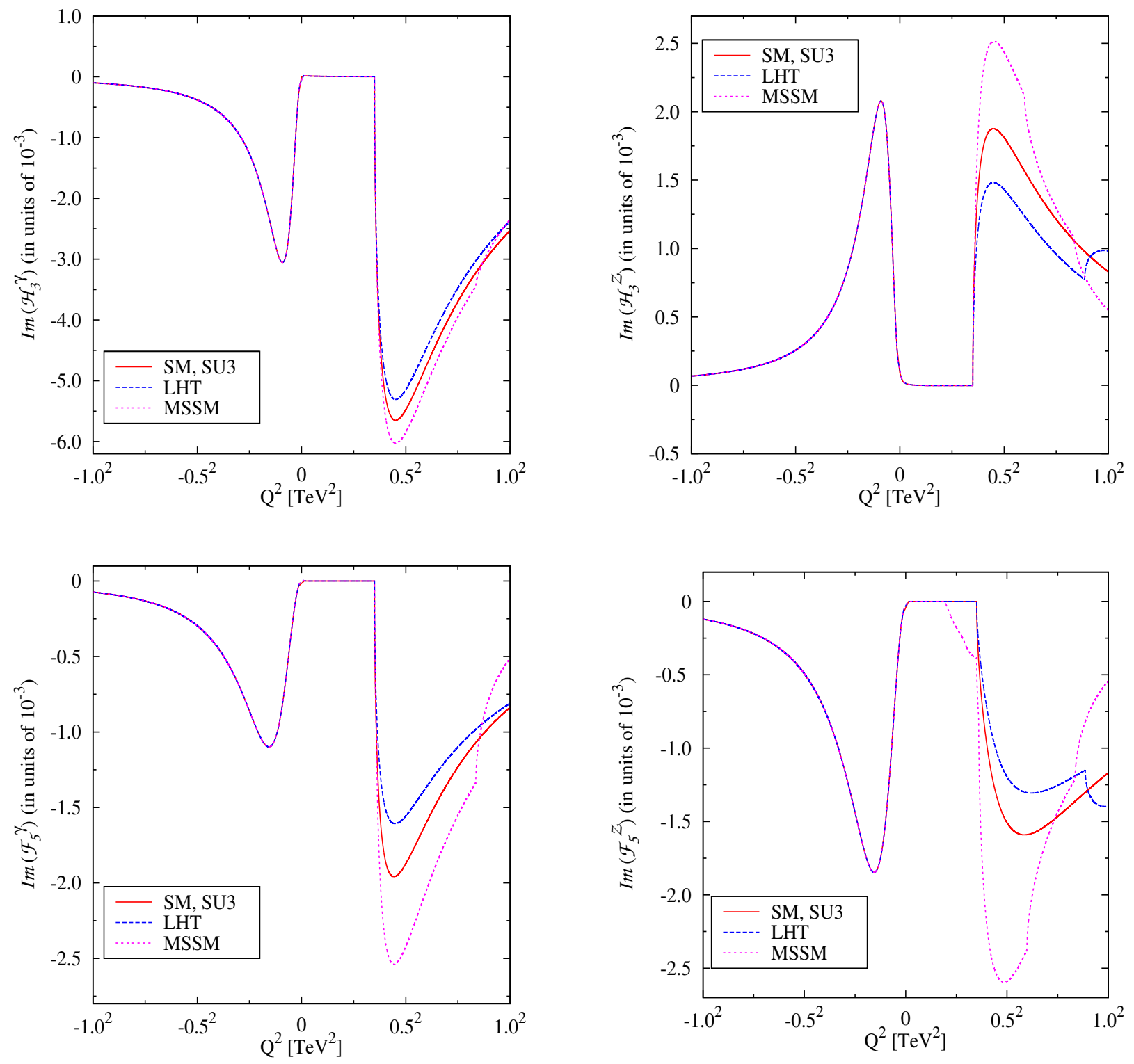

FIG. 4: $\sqrt{Q^{2}}$-variation of the imaginary parts of the couplings in various models for model parameter values $f=500 \mathrm{GeV}, r=1$ for LHT and $f=3 \mathrm{TeV}, t_{\beta}=3$ and masses of all heavy fermions, $M_{i}=2 \mathrm{TeV}$ for the SU(3) Model. MSSM parameters are as discussed in the text.

As discussed in Sec. I, the triple gauge boson couplings in the SM and MSSM have been already studied by various groups [4, 5]. Our results agree with the earlier results. However we have chosen parameter space defined by the reference point SPS1a' as mentioned in Sec. I. For this chosen point in the parameter space, the chargino masses are calculated to 
be $m_{\chi_{1}^{+}}=183.7 \mathrm{GeV}, m_{\chi_{2}^{+}}=415.4 \mathrm{GeV}$ and the neutralino masses are $m_{\chi_{1}^{0}}=94.8 \mathrm{GeV}$, $m_{\chi_{2}^{0}}=180.3 \mathrm{GeV}, m_{\chi_{3}^{0}}=401.9 \mathrm{GeV}$ and $m_{\chi_{4}^{0}}=411.8 \mathrm{GeV}$. In the MSSM there is a peak at $\sqrt{Q^{2}}=2 m_{\chi_{1}^{+}}$, which is very near to the $2 m_{t}$ SM peak for the special point chosen here. This results in the enhancement of the couplings at this point as can be seen from Figs. 3 and 4. This effect is more pronounced in the imaginary parts of the couplings. All couplings in MSSM show a threshold effect at $\sqrt{Q^{2}}=2 m_{\chi_{2}^{+}} \simeq 800 \mathrm{GeV}$ which is more pronounced in the real parts of $f_{5}^{\gamma}$ and $h_{3}^{Z}$ whereas in SM and Little Higgs Model there is no such effect upto 1 $\mathrm{TeV}$. Besides, in the MSSM new peaks appear at $m_{\chi_{1}^{+}}+m_{\chi_{2}^{+}}$and $2 m_{\chi_{2}^{+}}$. As mentioned before the neutralinos contribute only to $\mathcal{F}_{5}^{Z}$.

The effect of extra heavy fermions in the LHT Model is to decrease the threshold effects of the the SM whereas the particles in MSSM enhance it. The new threshold in the LHT at $\sqrt{Q^{2}}=m_{t}+M_{T_{+}}$and in the MSSM as mentioned above are opposite to each other but the magnitudes are comparable. As expected, the anomaly free SU(3) simple Model does not show any appreciably different behavior than the SM upto $\sqrt{Q^{2}}=1 \mathrm{TeV}$.

We have studied the trilinear neutral gauge boson couplings $\gamma^{\star} \gamma, Z^{\star} Z \gamma, \gamma^{\star} Z Z$ and $Z^{\star} Z Z$ involving one off-shell vector boson as a function of $Q^{2}$ in SM, MSSM and Little Higgs models. We have made theoretical prediction of these couplings for the model parameters which are constrained by the electro-weak precision measurements. The large s-channel contributions in the $Z Z$ and $Z \gamma$ production at the LHC due to the anomalous triple gauge boson couplings, could be the first indirect manifestation of new physics. The $Z Z$ cross-section will be measured at the start up of LHC with a significance of 4.8 at the $1 \mathrm{fb}^{-1}$ integrated luminosity and expected to suffer only a total of $12.9 \%$ uncertainties which include the PDF and QCD uncertainties [1]. However, a the precise measurement of the triple gauge boson couplings will only be possible with a $10 \mathrm{fb}^{-1}$ luminosity. Our analysis presented above allows us to confront and discriminate among various models considered here on the basis of these couplings.

The complementary study of the one-loop contribution to the triple charge - neutral gauge boson vertices $W^{+} W^{-} \gamma$ and $W^{+} W^{-} Z$ in the context of various Little Higgs Models with and without $\mathrm{T}$ parity is in process.

\section{Acknowledgments}

Authors would like to thank Prof. S. Rai Choudhury and Prof. Debajyoti Choudhury for fruitful discussions. The authors acknowledge the partial support from the Department of Science and Technology, India under grant SR/S2/HEP-12/2006 and the infra-structural support from the IUCAA Reference Center, Delhi. 


\section{APPENDIX A: FERMION 1 LOOP CONTRIBUTION TO CP EVEN COUPLINGS}

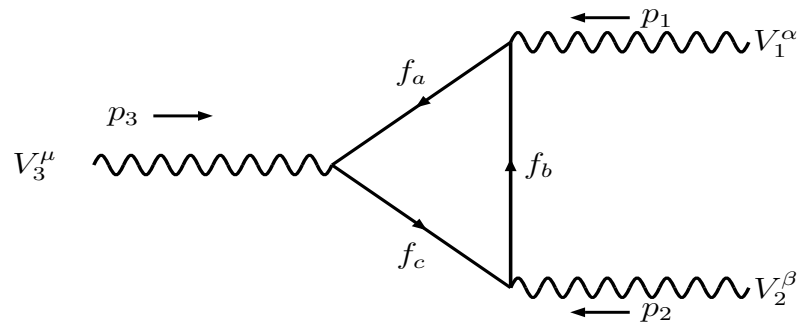

(a) Direct

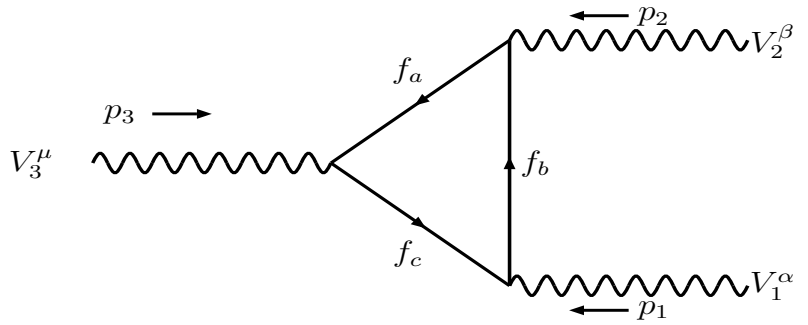

(b) Exchange

FIG. 5: Generic 1 loop diagrams contributing to CP conserving trilinear neutral gauge boson vertices.

Let us consider the vertex: $V_{1}\left(p_{1 \alpha}\right) V_{2}\left(p_{2 \beta}\right) V_{3}\left(p_{3 \mu}\right)$ where $V_{i} \equiv \gamma, Z$ and $p_{1}+p_{2}+p_{3}=0$. As for the $\mathrm{CP}$ conserving ones, to one-loop order, only the fermions in the theory contribute. In Fig. 5, we draw a generic direct and a exchange diagram contributing to this process. Denoting the fermion-gauge coupling by

$$
\mathcal{L}=\bar{f}_{i} \gamma_{\mu}\left[g_{L i j}^{\mathrm{V}} P_{L}+g_{R} \mathrm{~V} P_{R}\right] f_{j} \mathrm{~V}^{\mu}
$$

with $P_{L, R}=\left(1 \mp \gamma_{5}\right) / 2$. Throughout our analysis the momenta $p_{1}$ and $p_{2}$ denote same kind of bosons, say $\mathrm{V}_{1}^{\alpha}$ and $\mathrm{V}_{2}^{\beta}$, and momenta $p_{3}$ denotes $\mathrm{V}_{3}^{\mu}$ which is of the second kind of vector boson unlike the case of $Z Z Z$, where all are vector boson are same. It is useful to define the combinations in notation where $\mathrm{V} \equiv \mathrm{V}_{1} \equiv \mathrm{V}_{2}$.

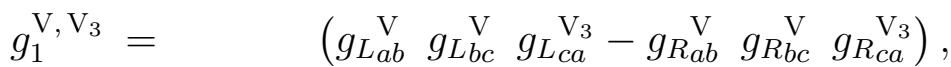

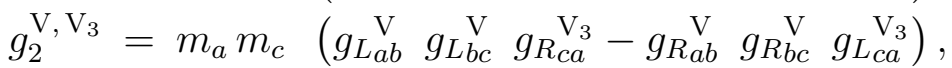

$$
\begin{aligned}
& g_{3}^{\mathrm{V}, \mathrm{V}_{3}}=m_{a} m_{b} \quad\left(\begin{array}{lllll}
g_{L a b}^{\mathrm{V}} & g_{R b c}^{\mathrm{V}} & g_{R} & \mathrm{~V}_{3} & -g_{R a b} \\
\mathrm{~V} & g_{L b c} & g_{L c a}^{\mathrm{V}}
\end{array}\right) \text {, }
\end{aligned}
$$

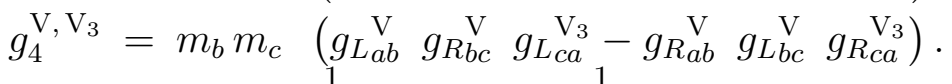

Here $m_{i}, i=a, b, c$, are the masses of the internal fermions $f_{i}$. The loop contribution with three distinct internal fermions $f_{a}, f_{b}, f_{c}$ having masses $m_{a}, m_{b}$ and $m_{c}$, respectively, corresponding to the direct diagram of Fig. 5(a) is proportional to $\epsilon_{\alpha \beta \mu \eta}$ and can be parameterized as ${ }^{[*]}$

$$
\begin{aligned}
\mathcal{A}_{a, b, c}^{\mathcal{D}}\left(\mathrm{V}, \mathrm{V}_{3} ; p_{1}, p_{2}, p_{3}\right) & =g_{1}^{\mathrm{V}, \mathrm{V}_{3}}\left[p_{3}^{2}\left(C_{11}+C_{21}\right)-p_{2}^{2}\left(C_{12}+C_{22}\right)-2 p_{1} \cdot p_{2}\left(C_{12}+C_{23}\right)\right] \\
& +\left(m_{a}^{2} g_{1}^{\mathrm{V}, \mathrm{V}_{3}}+g_{3}^{\mathrm{V}, \mathrm{V}_{3}}-g_{2}^{\mathrm{V}, \mathrm{V}_{3}}\right)\left(C_{11}+C_{0}\right)-g_{4}^{\mathrm{V}, \mathrm{V}_{3}} C_{11} .
\end{aligned}
$$

The contribution of exchange diagram with three distinct fermions $f_{a}, f_{b}, f_{c}$ as shown in the Fig. 5(b) having masses $m_{a}, m_{b}$ and $m_{c}$, respectively and proportional to the $\epsilon_{\alpha \beta \mu \eta}$ is

[*] We adopt the convention $\epsilon_{0123}=1$. Also we follow the notation of PV functions as given in Ref. [26] 
parameterized in terms of

$$
\begin{aligned}
\mathcal{A}_{a, b, c}^{\mathcal{E}}\left(\mathrm{V}, \mathrm{V}_{3} ; p_{1}, p_{2}, p_{3}\right)= & -\left(m_{a}^{2} g_{1}^{\mathrm{V}, \mathrm{V}_{3}}-g_{4}^{\mathrm{V}, \mathrm{V}_{3}}-g_{2}^{\mathrm{V}, \mathrm{V}_{3}}\right)\left(C_{11}-C_{12}\right)-g_{3}^{\mathrm{V}, \mathrm{V}_{3}}\left(C_{11}+C_{0}-C_{12}\right) \\
& -g_{1}^{\mathrm{V}, \mathrm{V}_{3}}\left[B_{023}+B_{123}-2 C_{24}+p_{1}^{2}\left(C_{12}+C_{21}\right)\right. \\
& \left.+\left(p_{2}^{2}+2 p_{1} \cdot p_{2}\right)\left(C_{22}-2 C_{23}+C_{21}\right)\right]
\end{aligned}
$$

In Eqs. A3 and $\mathrm{A} 4, B_{\mu j k}$ and $C_{\mu}, C_{\mu \nu}$ are respectively the 2-point and 3-point Passarino Veltman functions ([26]) defined as

$$
\begin{gathered}
B_{023}=B_{0}\left(p_{2}^{2}, m_{b}^{2}, m_{c}^{2}\right) ; B_{123}=B_{1}\left(p_{2}^{2}, m_{b}^{2}, m_{c}^{2}\right) \\
C_{0} ; C_{\mu} ; C_{\mu \nu}=C_{0} ; C_{\mu} ; C_{\mu \nu}\left(p_{3}^{2}, p_{2}^{2}, m_{a}^{2}, m_{c}^{2}, m_{b}^{2}\right)
\end{gathered}
$$

with

$$
\begin{aligned}
B_{0} ; B_{\mu} ; B_{\mu \nu}\left(p^{2}, m_{i}^{2}, m_{j}^{2}\right) & =\frac{1}{\iota \pi^{2}} \int d^{4} k \frac{1 ; k_{\mu} ; k_{\mu \nu}}{\left(k^{2}+m_{i}^{2}\right)\left[(k+p)^{2}+m_{j}^{2}\right]} \\
C_{0} ; C_{\mu} ; C_{\mu \nu}\left(p_{1}^{2}, p_{2}^{2}, m_{i}^{2}, m_{j}^{2}, m_{k}^{2}\right) & =\frac{1}{\iota \pi^{2}} \int d^{4} k \frac{1 ; k_{\mu} ; k_{\mu \nu}}{\left(k^{2}+m_{i}^{2}\right)\left[\left(k+p_{1}\right)^{2}+m_{j}^{2}\right]\left[\left(k+p_{1}+p_{2}\right)^{2}+m_{k}^{2}\right]}
\end{aligned}
$$

1. The loop amplitude for $\gamma^{\star} \gamma Z$ is

$$
\kappa \sum_{a=1}^{N_{f}} \sum_{b=1}^{N_{f}} \sum_{c=1}^{N_{f}} \mathcal{C}_{f}\left[\mathcal{A}_{a, b, c}^{\mathcal{D}}\left(\gamma, Z ; 0, \sqrt{Q^{2}}, m_{Z}\right)+\mathcal{A}_{a, b, c}^{\mathcal{E}}\left(\gamma, Z ; \sqrt{Q^{2}}, 0, m_{Z}\right)\right]
$$

where $N_{f}$ is the total number of flavors, $\mathcal{C}_{f}$ is the color factor of the fermion in the loop and $\kappa$ is the over all loop factor. Since electromagnetic interactions at two vertices forbids any flavor mixing, the above summation can be re-written as

$$
\begin{aligned}
\mathcal{H}_{3}^{\gamma}= & -\left(\frac{4 \pi \alpha_{\mathrm{em}}}{\left(2 \cos \theta_{W} \sin \theta_{W}\right)\left(16 \pi^{2}\right)}\right) \otimes \\
& \sum_{a=1}^{N_{f}} \mathcal{C}_{f}\left[\mathcal{A}_{a, a, a}^{\mathcal{D}}\left(\gamma, Z ; 0, \sqrt{Q^{2}}, m_{Z}\right)+\mathcal{A}_{a, a, a}^{\mathcal{E}}\left(\gamma, Z ; \sqrt{Q^{2}}, 0, m_{Z}\right)\right] .
\end{aligned}
$$

2. The loop amplitude for $\gamma^{\star} Z Z$ has one e.m. vertex which renders the mixing among the weak interaction eigenstates at the other two weak vertices giving

$$
\begin{aligned}
\mathcal{F}_{5}^{\gamma=} & \left(\frac{4 \pi \alpha_{\mathrm{em}}}{\left(2 \cos \theta_{W} \sin \theta_{W}\right)\left(16 \pi^{2}\right)}\right) \otimes \\
& \sum_{a=1}^{N_{f}} \sum_{b=1}^{N_{f}} \mathcal{C}_{f}\left[\mathcal{A}_{a, b, a}^{\mathcal{D}}\left(Z, \gamma ; m_{Z}, m_{Z}, \sqrt{Q^{2}}\right)+\mathcal{A}_{a, b, a}^{\mathcal{E}}\left(Z, \gamma ; m_{Z}, m_{Z}, \sqrt{Q^{2}}\right)\right]
\end{aligned}
$$


3. The loop amplitude for $Z^{\star} \gamma Z$ follows the same suit as the previous one with an additional change in the momentum assignment.

$$
\begin{aligned}
\mathcal{H}_{3}^{Z}= & \left(\frac{4 \pi \alpha_{\mathrm{em}}}{\left(2 \cos \theta_{W} \sin \theta_{W}\right)^{2}\left(16 \pi^{2}\right)}\right) \otimes \\
& \sum_{a=1}^{N_{f}} \sum_{b=1}^{N_{f}} \mathcal{C}_{f}\left[\mathcal{A}_{a, b, a}^{\mathcal{D}}\left(Z, \gamma ; m_{Z}, \sqrt{Q^{2}}, 0\right)+\mathcal{A}_{a, b, a}^{\mathcal{E}}\left(Z, \gamma ; \sqrt{Q^{2}}, m_{Z}, 0\right)\right]
\end{aligned}
$$

4. The loop amplitude for $Z^{\star} Z Z$ allow weak mixing at all vertices.

$$
\begin{aligned}
\mathcal{F}_{5}^{Z}= & \left(\frac{4 \pi \alpha_{\mathrm{em}}}{\left(2 \sin \theta_{W} \cos \theta_{W}\right)^{3}\left(16 \pi^{2}\right)}\right) \otimes \\
& \sum_{a=1}^{N_{f}} \sum_{b=1}^{N_{f}} \sum_{c=1}^{N_{f}} \mathcal{C}_{f}\left[\mathcal{A}_{a, b, c}^{\mathcal{D}}\left(Z, Z ; m_{Z}, m_{Z}, \sqrt{Q^{2}}\right)+\mathcal{A}_{a, b, c}^{\mathcal{E}}\left(Z, Z ; m_{Z}, m_{Z}, \sqrt{Q^{2}}\right)\right]
\end{aligned}
$$

\begin{tabular}{|c|c|c|c|c|}
\hline \multirow[t]{2}{*}{ Vertex } & \multicolumn{2}{|c|}{ LHT Model } & \multicolumn{2}{|l|}{ SU(3) simple group } \\
\hline & $g_{L}$ & $g_{R}$ & $g_{L}$ & $g_{R}$ \\
\hline $\begin{array}{l}\qquad \bar{q}_{i} Z q_{i} \\
(\text { for } i=1-5) \\
\text { i.e. all SM quarks except top }\end{array}$ & $2\left(T_{3}^{i}-Q^{i} s_{w}^{2}\right)$ & $-2 Q^{i} s_{w}^{2}$ & $2\left(T_{3}^{i}-Q^{i} s_{w}^{2}\right)$ & $-2 Q^{i} s_{w}^{2}$ \\
\hline $\begin{array}{l}\qquad \bar{l}_{i} Z l_{i} \\
\quad(\text { for } i=1-6) \\
\text { i.e. all SM leptons }\end{array}$ & $2\left(T_{3}^{i}-Q^{i} s_{w}^{2}\right)$ & $-2 Q^{i} s_{w}^{2}$ & $2\left(T_{3}^{i}-Q^{i} s_{w}^{2}\right)$ & $-2 Q^{i} s_{w}^{2}$ \\
\hline $\bar{t} Z t$ & $1-\frac{4}{3} s_{w}^{2}-x_{L}^{2} \frac{v^{2}}{f^{2}}$ & $-\frac{4}{3} s_{w}^{2}$ & $1-\frac{4}{3} s_{w}^{2}-x_{L}^{2} \frac{v^{2}}{f^{2}}$ & $-\frac{4}{3} s_{w}^{2}$ \\
\hline $\bar{T} Z t$ & $x_{L} \frac{v}{f}$ & 0 & $\frac{1}{2 \sqrt{2}\left(r^{2}+t_{\beta}^{2}\right)} s_{2 \beta}\left(1+t_{\beta}^{2}\right)\left(r^{2}-1\right) \frac{v}{f}$ & 0 \\
\hline $\bar{T} Z T$ & $x_{L}^{2} \frac{v^{2}}{f^{2}}-\frac{4}{3} s_{w}^{2}$ & $-\frac{4}{3} s_{w}^{2}$ & 0 & 0 \\
\hline $\begin{array}{l}\bar{D}_{i} Z d_{i} \\
i=1,2\end{array}$ & $\times x$ & $\times \times$ & $\frac{1}{\sqrt{2} t_{\beta}} \frac{v}{f}$ & 0 \\
\hline $\begin{array}{c}\bar{N}_{i} Z \nu_{i} \\
i=1,2,3\end{array}$ & $\times \times$ & $\times x$ & $-\frac{1}{\sqrt{2} t_{\beta}} \frac{v}{f}$ & 0 \\
\hline
\end{tabular}

TABLE IV: Relevant Couplings of fermions with Z-boson in units of $g / 2 c_{w}$ in the Littlest Higgs Model with T-parity (LHT) and SU(3) simple group model with anomaly-free embedding. Note that $T$ denotes the T-even heavy top quark in case of LHT and is the heavy top in case of SU(3) Model. Here $x_{L}=\frac{1}{1+r^{-2}}$ with $r=\lambda_{1} / \lambda_{2}$. 
[1] G. L. Bayatian et al. [CMS Collaboration], J. Phys. G 34, 995 (2007).

[2] A. Djouadi et al. [ILC Collaboration], arXiv:0709.1893 [hep-ph].

[3] G. Gounaris et al., arXiv:hep-ph/9601233; E. N. Argyres, A. B. Lahanas, C. G. Papadopoulos and V. C. Spanos, Phys. Lett. B 383, 63 (1996) [arXiv:hep-ph/9603362]; E. N. Argyres, G. Katsilieris, A. B. Lahanas, C. G. Papadopoulos and V. C. Spanos, Nucl. Phys. B 391, 23 (1993). A. Arhrib, J. L. Kneur and G. Moultaka, Phys. Lett. B 376, 127 (1996) [arXiv:hep-ph/9512437].

[4] G. J. Gounaris, J. Layssac and F. M. Renard, Phys. Rev. D 61, 073013 (2000) [arXiv:hep$\mathrm{ph} / 9910395]$.

[5] D. Choudhury, S. Dutta, S. Rakshit and S. Rindani, Int. J. Mod. Phys. A 16, 4891 (2001) [arXiv:hep-ph/0011205].

[6] R. Armillis, C. Coriano and M. Guzzi, JHEP 0805, 15 (2008) [arXiv:0711.3424 [hep-ph]].

[7] U. Baur and E.L. Berger, Phys. Rev. D47, 4889 (1993); U. Baur, T. Han and J. Ohnemus, Phys. Rev. D57, 2823 (1998); U. Baur and D. Rainwater, Phys. Rev. D52, 112011 (2000) and references therein.

[8] J. Deng, FERMILAB-THESIS-2008-37, (2008); T. Aaltonen et al. [CDF Collaboration], Phys. Rev. D 76, 111103 (2007) [arXiv:0705.2247 [hep-ex]].

[9] J. Abdallah et al. [DELPHI Collaboration], Eur. Phys. J. C 51, 525 (2007) [arXiv:0706.2741 [hepex]]; M. Acciarri et al. [L3 Collaboration], Phys. Lett. B 489 (2000) 55 [arXiv:hep-ex/0005024].

[10] N. Arkani-Hamed, A. G. Cohen, E. Katz, A. E. Nelson, T. Gregoire and J. G. Wacker, JHEP 0208, 021 (2002) [arXiv:hep-ph/0206020].

[11] N. Arkani-Hamed, A. G. Cohen, E. Katz and A. E. Nelson, JHEP 0207, 034 (2002) [arXiv:hep$\mathrm{ph} / 0206021]$.

[12] I. Low, W. Skiba and D. Tucker-Smith, Phys. Rev. D 66, 072001 (2002) [arXiv:hep-ph/0207243]; S. Chang and J. G. Wacker, Phys. Rev. D 69, 035002 (2004) [arXiv:hep-ph/0303001];

S. Chang, JHEP 0312, 057 (2003) [arXiv:hep-ph/0306034].

[13] W. Skiba and J. Terning, Phys. Rev. D 68, 075001 (2003) [arXiv:hep-ph/0305302].

[14] D. E. Kaplan and M. Schmaltz, JHEP 0310, 039 (2003) [arXiv:hep-ph/0302049].

[15] M. Schmaltz, JHEP 0408, 056 (2004) [arXiv:hep-ph/0407143].

[16] C. Csaki, J. Hubisz, G. D. Kribs, P. Meade and J. Terning, Phys. Rev. D 68, 035009 (2003) [arXiv:hep-ph/0303236]; J. L. Hewett, F. J. Petriello and T. G. Rizzo, JHEP 0310, 062 (2003) [arXiv:hep-ph/0211218].

[17] R. Barbieri, A. Pomarol, R. Rattazzi and A. Strumia, Nucl. Phys. B 703, 127 (2004) [arXiv:hepph/0405040]; Z. Han and W. Skiba, Phys. Rev. D 72, 035005 (2005) [arXiv:hep-ph/0506206].

[18] G. Marandella, C. Schappacher and A. Strumia, Phys. Rev. D 72, 035014 (2005) [arXiv:hep$\mathrm{ph} / 0502096]$. 
[19] H. C. Cheng and I. Low, JHEP 0309, 051 (2003) [arXiv:hep-ph/0308199].

[20] J. Hubisz and P. Meade, Phys. Rev. D 71, 035016 (2005) [arXiv:hep-ph/0411264]; J. Hubisz, S. J. Lee and G. Paz, JHEP 0606, 041 (2006) [arXiv:hep-ph/0512169]; J. Hubisz, P. Meade, A. Noble and M. Perelstein, JHEP 0601, 135 (2006) [arXiv:hep-ph/0506042].

[21] S. R. Choudhury, N. Gaur, A. Goyal and N. Mahajan, Phys. Lett. B 601, 164 (2004) [arXiv:hepph/0407050]; M. Blanke, A. J. Buras, A. Poschenrieder, C. Tarantino, S. Uhlig and A. Weiler, JHEP 0612, 003 (2006) [arXiv:hep-ph/0605214]; M. Blanke, A. J. Buras, A. Poschenrieder, S. Recksiegel, C. Tarantino, S. Uhlig and A. Weiler, JHEP 0701, 066 (2007) [arXiv:hepph/0610298]; A. Goyal, Mod. Phys. Lett. A 21, 1931 (2006); S. R. Choudhury, N. Gaur and A. Goyal, Phys. Rev. D 72, 097702 (2005) [arXiv:hep-ph/0508146]; M. Blanke, A. J. Buras, B. Duling, A. Poschenrieder and C. Tarantino, JHEP 0705, 013 (2007) [arXiv:hep-ph/0702136].

[22] K. Hagiwara, R. D. Pecci, D. Zepanfeld and K. Hikasa, Nucl. Phys. B282, 253 (1987).

[23] T. Han, H. E. Logan and L. T. Wang, JHEP 0601, 099 (2006) [arXiv:hep-ph/0506313].

[24] R. Barbieri, A. Pomaral, R. Rattazi and A. Strumia, Nucl. Phys. B 703, 127 (2004).

[25] J. A. Aguilar-Saavedra et. al., EPJC (2006) arXiv:hep-ph/0511344V1

[26] G. Passarino and M. Veltman, Nucl. Phys. B 160, 151 (1971). 